\title{
Estimulación y Enseñanza de Enfoque Intercultural en el Mundo Indígena; Colta - Sicalpa Ecuador Ñuka yachag, ñukanchipak yachag, tukaypak yachag. Mi educación, nuestra educación y educación para todos \\ Stimulation and Teaching of Intercultural Approach in the Indigenous World; Colta - Sicalpa Ecuador
}

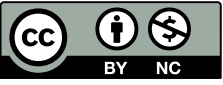

\author{
Bacilio Segundo Pomaina Pilamunga ${ }^{1}$ \\ bacilio_segundo@hotmail.com \\ Ana Lorena Lema Paltan ${ }^{2}$
}

Recibido: 1/07/2017, Aceptado: 1/09/2017

\section{RESUMEN}

La estimulación intercultural en el mudo indígena, se concibe como las metodologías de enseñanza y aprendizaje, el cual se evidencia en el ritual, el recorrido, la oralidad, la minga, el arte y la simbología, en estos procesos se visualiza el valor de la dualidad, tripartición y la cuatripartición. El presente trabajo es un estudio se realizado en las comunidades de Ocpote del cantón Colta provincia de Chimborazo Ecuador; cuyo trabajo implico compilación, sistematización y validación con la población local, en la cual se diseñó una cartilla de estimulación de enfoque intercultural. El documento formativo familiar y comunitario aborda el aspecto educativo comunitario, espiritual, histórico, cultural, social, y además precisa las técnicas y las metodologías de enseñanza indígena tanto la lengua y como la cultura. En el contexto indígena la formación del ser humano no empieza desde el nacimiento del ser humano sino de mucho más antes de la fecundación, es decir, desde el enamoramiento y sus entornos. La cartilla ha sido aplicada en el entorno educativo y familiar durante estos años, previo a la presentación, en el Simposio internacional de lenguas y culturas indígenas de América Latina. Bajo estos enfoques epistémicos y metodológicos, la estimulación y la enseñanza del enfoque indígena intercultural, es el camino para recuperar la dignidad de los seres humanos basados en sabiduría, conocimientos y saberes locales. El presente artículo propone dar respuestas a las siguientes interrogantes. ¿Qué es estimulación intercultural en el mundo indígena? ¿Cuáles son las metodologías de enseñanza indígena? ¿Cuáles son los espacios de educación indígena? ¿Qué áreas y niveles de la educación indígena integra?

Palabras clave: Educación y estimulación intercultural, técnicas y metodologías de enseñanza indígena

\footnotetext{
${ }^{1}$ Docente de la Universidad de Técnica de Machala. Ecuador

${ }^{2}$ Docente del Hospital General Docente de Latacunga. Ecuador
} 


\title{
II Jornada de Investigación Universidad Tecnológica Empresarial de Guayaquil
}

\begin{abstract}
The intercultural stimulation in the indigenous mudo, is conceived as the teaching and learning methodologies, which is evidenced in the ritual, the route, the orality, the minga, the art and the symbolism, in these processes the value of the duality, tripartition and quadripartition. The present work is a study carried out in the communities of Ocpote of the canton Colta province of Chimborazo Ecuador; whose work involved compilation, systematization and validation with the local population, in which a booklet for stimulating an intercultural approach was designed. The family and community training document address the educational, community, spiritual, historical, cultural, and social aspect, and also requires indigenous teaching techniques and methodologies, both language and culture. In the indigenous context, the formation of the human being does not start from the birth of the human being but of much more before the fertilization, that is to say, from the infatuation and its surroundings. The booklet has been applied in the educational and family environment during these years, prior to the presentation, in the International Symposium of indigenous languages and cultures of Latin America. Under these epistemic and methodological approaches, the stimulation and teaching of the intercultural indigenous approach is the way to recover the dignity of human beings based on wisdom, knowledge and local knowledge. This article proposes to give answers to the following questions. What is intercultural stimulation in the indigenous world? What are the indigenous teaching methodologies? What are the spaces for indigenous education? What areas and levels of indigenous education do you integrate?
\end{abstract}

Keywords: Education and intercultural stimulation, techniques and methodologies of indigenous education

\section{Introducción}

La presente investigación se refiere a la estimulación intercultural en el mundo indígena, que se puede definir como un sistema inicial de enseñanza y aprendizaje indígena, basada en los saberes ancestrales. La característica de este sistema es muy poco conocido en el ámbito educativo oficial ecuatoriano, pero es una práctica actual que aplican las comunidades indígenas del país; a raíz de esta práctica ha existido varias interpretaciones, cuestionamientos, hasta inclusive considerar como una práctica atentatoria a los derechos de la niñez y a dolencia.

Bajos estas primicias se desprende varias inquietudes entre ello, existe estimulación intercultural, cuales las metodologías de enseñanza y aprendizaje indígena, las prácticas de enseñanza y aprendizaje indígena atentan los derechos de la niñez y a dolencia. Con el propósito de conocer estas inquietudes, se desarrolla la presente investigación, ya que es un interés comunitario trascendental para la reivindicación de sus saberes y conocimientos. Por otra parte, busca sistematizar criterios, métodos, técnica y valores, que fortalezcan la perspectiva intercultural del saber para la comunidad.

El trabajo se sustente en la investigación cualitativa, basada en la fenomenología, hermenéutica indígena, principalmente de la investigación-acción participativa. El propósito de investigación tuvo los siguientes objetivos: 


\section{Jornada de Investigación Universidad Tecnológica Empresarial de Guayaquil}

- Recopilar los saberes de estimulación intercultural a través de la oralidad en los actores claves de la Comunidad Ocpote Los Ángeles.

- Sistematizar los saberes de estimulación intercultural mediante métodos participativos de validación para generar directrices de orientación comunitaria.

- Evidenciar la practica saberes de estimulación intercultural en los padres, madres y familiares de la comunidad a través de la observación, para determinar aspectos de difusión.

El presente estudio educativo cultural aborda las siguientes temáticas: Contexto territorial y cultural; nociones de estimulación de enfoque intercultural; fundamentos de estimulación intercultural; técnicas, espacios, áreas y niveles de educación indígena.

\section{Metodología}

La enseñanza de los saberes ancestrales desde el enfoque intercultural nos convoca a los actores educativos a establecer preceptos, nociones y tácticas que permitan la implementación del estado plurinacional en el Ecuador. La presente investigación se sustenta en un método de investigación colectivista de grupo focal, se fundamenta en la epistemología cualitativa, es un estudio de nuestra no probabilístico. Para el desarrollo del trabajo, se procede a una serie de entrevistas a taitas (sabios), mamas (sabias), docentes, niños y niñas de la Comunidad Ocpote Los Ángeles; el ítem de las entrevistas no tuvo número definido, pero si el perfil del contenido de la discusión, entre ello: estimulación intercultural, fundamentos, métodos de enseñanza, etapas, técnicas, áreas y niveles de educación indígena.

Para la selección de los entrevistados, se estableció procedimientos, criterios y perfiles a cumplir antes de la aplicar las entrevistas, a; taitas (sabios), mamas (sabias), docentes, niños y niñas de la Comunidad.

Criterios de selección. -

- Taitas (sabios), mamas (sabias), destacada en la enseñanza comunitaria.

- Docentes destacadas y destacados en la enseñanza comunitaria.

- Niños y niñas de la Comunidad que practicas la enseñanza de los saberes comunitarios.

- Padres y madres, destacada en la enseñanza familiar, cultural y comunitaria.

El estudio del presente trabajo es nuestra no probabilística; por lo que no posee nuestra ni el universo del análisis; para la sistematización de criterios y preceptos se priorizó y seleccionó de acuerdo a los el ítem de las entrevistas, sus criterio y testimonios de propios actores.

\section{Contexto territorial y cultural}

La comunidad de Ocpotes Los Ángeles, se encuentran localizada en la república del Ecuador, provincia de Chimborazo, cantón Colta, parroquia de Sicalpa, junto a la meseta de la cordillera occidental; a $6 \mathrm{~km}$ desde la cabecera cantonal (Riobamba), es una comunidad indígena de acontecimientos históricos, sociales y culturales.

La comunidad es descendiente del pueblo Sicalpa, un pueblo que formaba parte de la nación Puruhá que constituía ancestralmente un Ayllu Llacta (pueblo) y ayllu 


\section{Jornada de Investigación Universidad Tecnológica Empresarial de Guayaquil}

(familias), bajo una estructura política, social, jurídica, económica, cultural, filosófica y espiritual. Durante nuestra investigación visitamos Llactakuna (pueblo) y Ayllukuna (familias) del pueblo Sicalpa, en la cual comprobamos que aún existe la indumentaria y la dialéctica en los abuelos y abuelas de las comunidades de Ocpotes. Lo más relevante es que aún se conserva en la oralidad los nombres de llacta, ayllu, cerros, apellidos y límites naturales, muy identificados con la indumentaria y la dialéctica de cada Ayllu Llacta (pueblo).

Bajo estas estructuras territoriales se configuran los Llacatas (Pueblo) de Ocpotes con sus propias dinámicas jurídicas, políticas, culturales y espirituales. Al principio el denominado Ocpote Llacta estaba conformado en un solo territorio, con la creación de la comunidad en las décadas del 70, se desestructuró y se dividió en las siguientes comunidades: Ocpote Guáyalo, Ocpote Yavirag, Ocpote Concepción, Ocpote La Merced, Ocpote San Vicente, Ocpote Villa María, Ocpote Centro, Ocpote San Luis, Ocpote Rumipamba, Ocpote Los Angeles, Ocpote Tablarumi y Ocpotillo Chico.

La palabra OCPOTE (Balla, 2015) proviene del significado indígena, surge por la unión dedos significados: OCPO = ocpocho, es una tuna de espino que se encuentra en los cercos y chaparros; POTE = poteg, es un arbusto pequeño tradicional de la comunidad con hojas largas y flores morados. La traducción al español significa Llacata (Pueblo) de ESPINO y ARBUSTO.

Según la vivencia indígena, los habitantes de esta comunidad posen vestimentas muy distintas a otras zonas geográficas. Los hombres usaban camisas de tela manga larga, pantalón de basta campana, en ocasiones de frio se ponían samaro (pantalón de lana de borrego), se cangan azial (un látigo de cuero) como símbolo de mando o poder, el poncho kusma (kusma es también un tipo de poncho delgado, usado debajo del principal) de color azul o rojo.

En cambio, las mujeres convierten dos anacos (manto negro de tela que cubre desde la cintura hasta los tobillos) largos en un camisón de color negro que cubre desde el cuello hasta las rodillas. A esta vestimenta le acompañaban la bayeta (manto de tela rojo o verde que cubre la espalda y pecho), el anaco, el chagalli (manto que cubre desde el ombligo hasta los tobillos por encima del anaco), las orejeras (collares de perla que se ponen en la parte inferior del oído), las fajas de kawiña (cinta gruesa de color tejida a mano que se envuelve en varias vueltas alrededor de la cintura), el tupo (imperdible grueso que abrocha la bayeta), la macana (manto blanco de cargar el almuerzo), la cinta de amarrar el cabello, amanillas (mullos de perlas delgadas que se colocan en las manos) y los collares (mullos de perlas gruesas que se colocan en el cuello) de varios colores.

La indumentaria de Ocpotes, se diferencia en la forma de vestir y el dialecto, los colores de mayor preferencia de los Ocpotes es negro, rojo, azul y lacre, el dialecto que diferencia a Opotes es el uso de terminación fonética shi en las expresiones con acento fuerte al término de la palabra. Ver fotografía 1. 


\section{Jornada de Investigación Universidad Tecnológica Empresarial de Guayaquil}

\section{Fotografía 1. Indumentaria de Ocpotes}
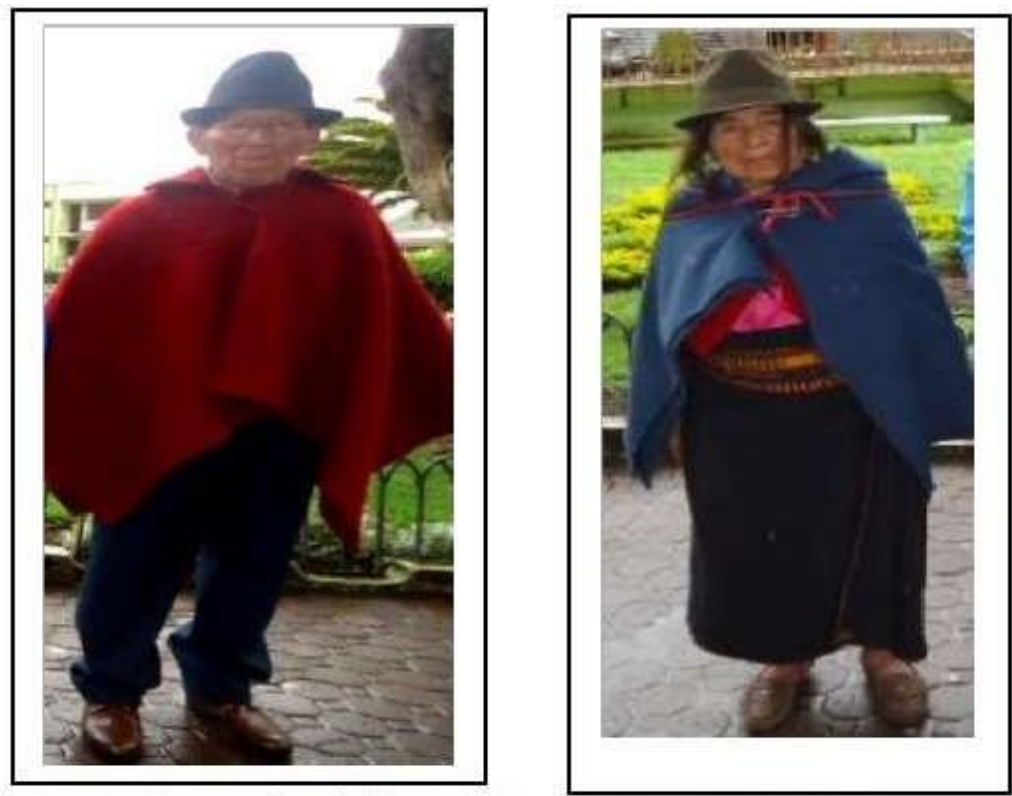

Fotografía 05/08/2010: Manuel y María de Ocpotes

Fotografía 05/08/2010: Manuel y María de Ocpotes

\section{Nociones de estimulación de enfoque intercultural}

Es evidente que en el tema de la estimulación en la cotidianidad existen diferentes preceptos, enfoques y modelos para su aplicación, a continuación, algunas perspectivas de estimulación según autores:

Para el autor Orlando Terré, presidente de la Asociación Mundial de Educación; la estimulación es el conjunto de medios, técnicas, y actividades, aplicada en forma sistémica y secuencial, desde el nacimiento hasta los seis años, con el objetivo de desarrollar al máximo sus capacidades cognitivas, físicas y psíquicas.

En cambio, para Dr. Alejandro Medina Salas de la Revista Mexicana de Medicina Física y Rehabilitación; se define como un conjunto de acciones que potencializan al máximo las habilidades físicas, mentales y psicosociales del niño, mediante la estimulación repetitiva, continua y sistematizada.

Mientras la revista Guía de estimulación temprana escrita por editores ADRA de Perú; lo define con un conjunto de ejercicios, juegos y otras actividades que se les brinda a los niños y niñas de manera repetitiva en sus primeros años de vida, con el objetivo de desarrollar al máximo sus capacidades físicas, emocionales, sociales y de aprendizaje.

Con los preceptos expuestos; a continuación, la definición desde el contexto indígena. La estimulación intercultural (Guacho, 2015) es un método de formación para la vida al ser humano; esto implica conexión con la familia, la comunidad, la naturaleza y los valores cultural, en un vínculo integral irrenunciable entre comunidad humana, natural, ancestral y divino, no es solo educar en el humanismo a los individuos, sino en contexto integral de vida. 


\section{Jornada de Investigación Universidad Tecnológica Empresarial de Guayaquil}

Comunidad divina, representado por el Pachakamak (Dios).

Comunidad de ancestros, representado por ancestros fallecidos.

Comunidad natural, representada por los seres materiales de la tierra (flora, fauna, minerales, manantiales, entre otros).

Comunidad humana, representada por la sociedad vigente (seres humanos o habitantes).

En algunos taitas (sabios) (Balla, 2015), se considera como una pedagogía, un camino para recuperar la dignidad de los seres humanos basados en la sabiduría andina, conocimientos, y la ciencia comunitaria. Se centra en la enseñanza para la vida, a través de ver, oír, sentir y actuar, la cual permite el fortalecimiento de modos de vida local, activando la participación y la toma de conciencia cultural; a esto se suma la lucha por reivindicación de los derechos colectivos y sus saberes.

Desde estas dimensiones culturales, se precisa que la estimulación intercultural, se inicia desde el enamoramiento de las parejas y la fase se concluye cuando el niño o la niña adquieren su autonomía, posteriormente inicia otra fase de educación y aprendizaje, es decir la educación al nuevo ser humano, no se concluye solo en la estimulación, la enseñanza se trasciende hasta convertirse taita (sabio), para lo cual, hay métodos, áreas y niveles de educación andina.

La enseñanza de los padres y madres indígenas, se centra en difundir la sabiduría andina, compartir la vivencia y valorar la complementariedad (lo que sabe el padre o la madres, lo transmite y su multiplicación genera un saber complementario), la reciprocidad (se aprende y se enseña al mismo tiempo) y la proporcionalidad (un saber para cada quien); estos saberes cotidianos permite retornar a los nuevos tiempos, donde la metodología, los espacios y el área de formación andina tiene un lugar y un espacio, aun latente en los tiempos de posmodernidad, globalización y homogenización educativa.

La educación de saberes (Mullo, 2015) no solo se trata de hacer educación secular, en los pueblos indígenas, a más de recibir esa educación, también hay que fortalecer las habilidades y aptitudes colectivas; la familia, la comunidad y la naturaleza, que son ejes primordiales de enseñanza y aprendizaje colectivo. Con estos preceptos epistémicos, se puede notar que existen a diferencia entre la estimulación convencional y la estimulación intercultural, pero cuál es la diferencia, a continuación, los fundamentos, etapas y las técnicas de estimulación intercultural.

\section{Fundamento epistemológico de estimulación en el mundo indígena}

La estimulación intercultural (Cachiguango, 2007), se basa en una concepción integral, "holística", del universo, la naturaleza como madre tierra en donde todos los seres son parte, incluyendo los humanos. El ser humano pertenece a la naturaleza y la naturaleza al ser humano; para evidenciar esta conexión cósmica, es indispensable la conexión cósmica de la Chacana denominado también Cruz del Sur o Cruz Cuadrada que constituye la síntesis del sistema de leyes de formación y composición simbólica de la iconología geométrica andina, asimismo, es un concepto astronómico ligado a los saberes de los pueblos indígenas, con el yachag (saber), munay (amar), ruray (hacer) y munay (poder). (Ver gráfico). 


\section{Jornada de Investigación Universidad Tecnológica Empresarial de Guayaquil}

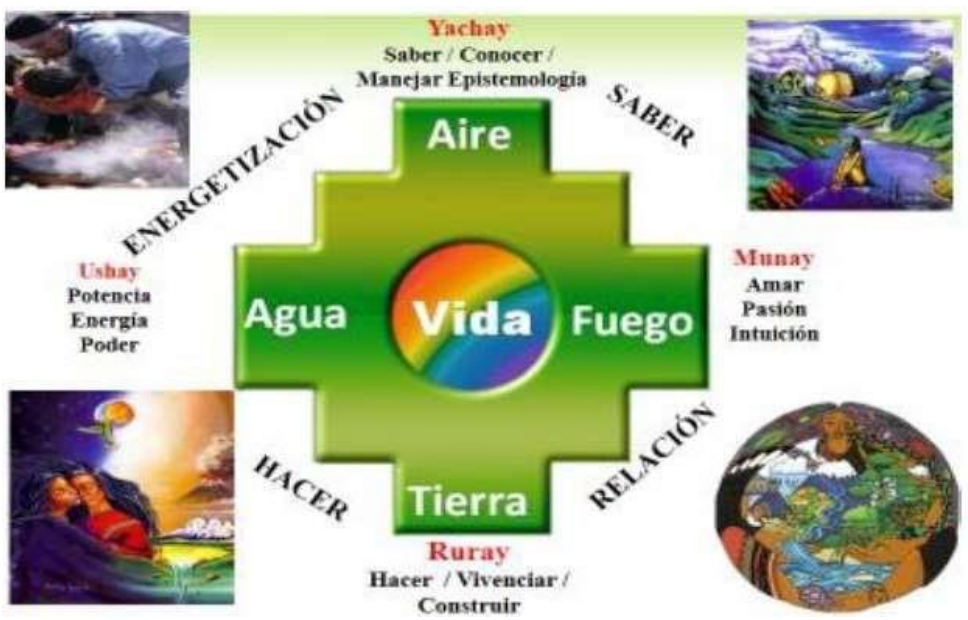

Gráfico 1. Iconología geométrica andina

Fuente: elaboración propia

La estimulación en el mundo indígena (Cepeda, 2015) está ligada a la enseñanza y formación para la VIDA; para cual contienen las siguientes fases: energetización, saber diferenciar, relación con el entorno y saber hacer. En cada una de estas fases está el yachag (saber), el munay (amar), el ruray (hacer) y ushay (poder), la cual se logra a través de conexión con los elementos de la vida (aire, agua, fuego y tierra).

La fase de energetización: corresponde a la estimulación inicial, la cual se inicia con la estimación en el vientre materno, el manteo (encuadernación de embarazo), el parto intercultural, el maito (envoltura) y el ritual de intensificación del nuevo ser. A lo largo de esta fase el niño o la niña está acompañado de actividades, limpias energéticas, de acuerdo al requerimiento y al contexto socio cultural. (Fotografía 2).

\section{Imagen 1 Contexto socio cultural}

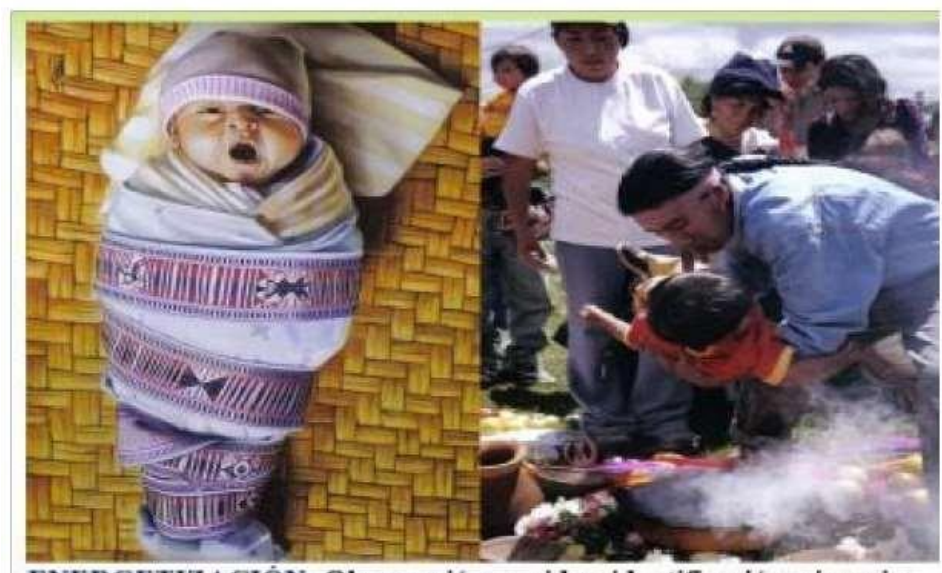

ENERGETIZACIÓN: Observación, sonidos, identifícación, vivencia.

Imagen editada: Bacilio Pomaina Pilamunga 


\section{Jornada de Investigación Universidad Tecnológica Empresarial de Guayaquil}

La fase de saber diferenciar: después del nacimiento del nuevo ser humano, se inicia la otra fase, que está a cargo del padre, madre y sus familiares; quienes enseñan a través de consejos, cantos, simulaciones, a diferencias, cosas, aspectos y valores culturales para la vida.

Esta etapa se considera los primeros años de nacimiento, al impartir estos saberes, el niño o la niña, se siente preparado para la siguiente fase. Ver gráfico.

\section{Imagen 3 Preparación infantil}

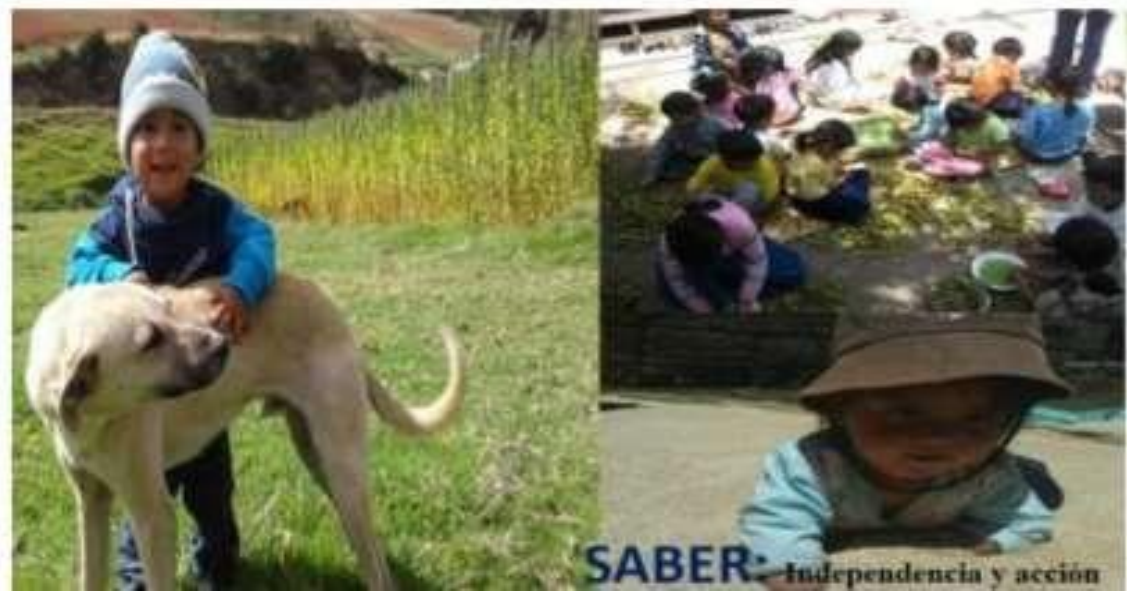

Imagen editada: Bacilio Pomaina Pilamunga

La fase de relación con el entorno: dependiendo del desarrollo de las capacidades cognitivas, físicas y psíquicas, se considera a partir de un año en adelante; en esta fase el niño o la niña está preparado para relacionarse con el entorno de los elementos naturales: aire, agua, fuego y tierra. En el cual diferencia, aplica o relaciona, con los valores, códigos y preceptos adquiridos en las fases anteriores. Ver imagen.

\section{Imagen 4. La relación con el entorno}

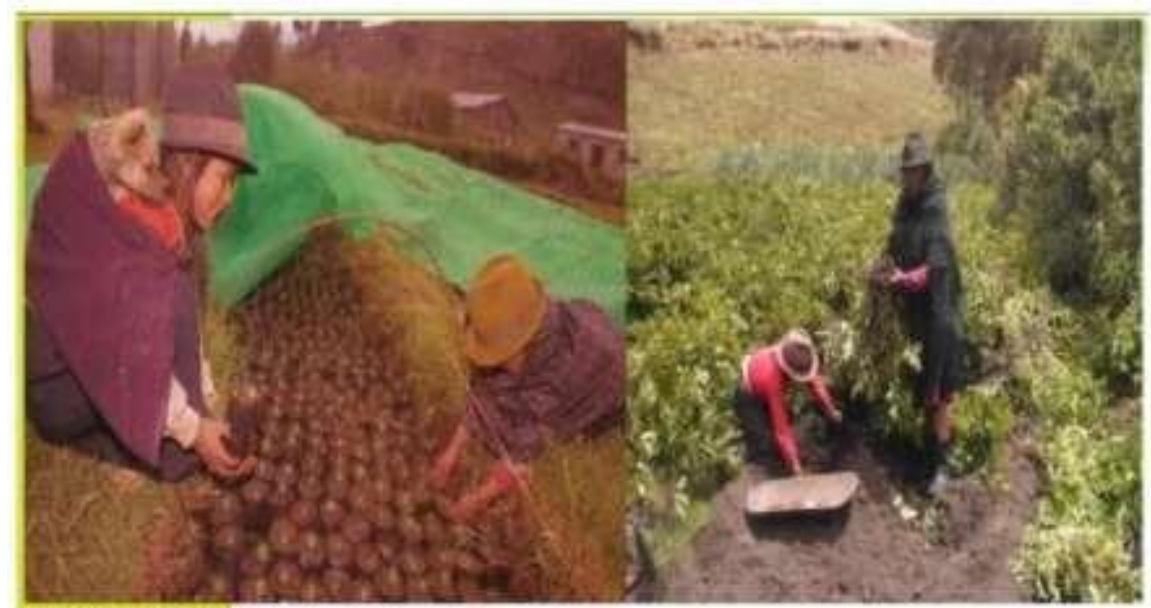

RELLACION: Imitación y vivencia.

Imagen editada: Bacilio Pomaina Pilamunga 


\section{Jornada de Investigación Universidad Tecnológica Empresarial de Guayaquil}

La fase de saber hacer: generalmente esta etapa se desprende a partir de 2 a 3 años de nacimiento, en el cual el niño o la niña, pone en práctica la enseñanza de los saberes de sus padres, familiar y comunidad. Esta esta etapa se ve con precisión la simulación, el emprendimiento, el liderazgo, y sobre todo los preceptos culturales de su pueblo; no se concluye en esta fase la enseñanza de los saberes, se trasciende a la formación en áreas y niveles. Ver imagen.

\section{Imagen 4. Saber hacer}

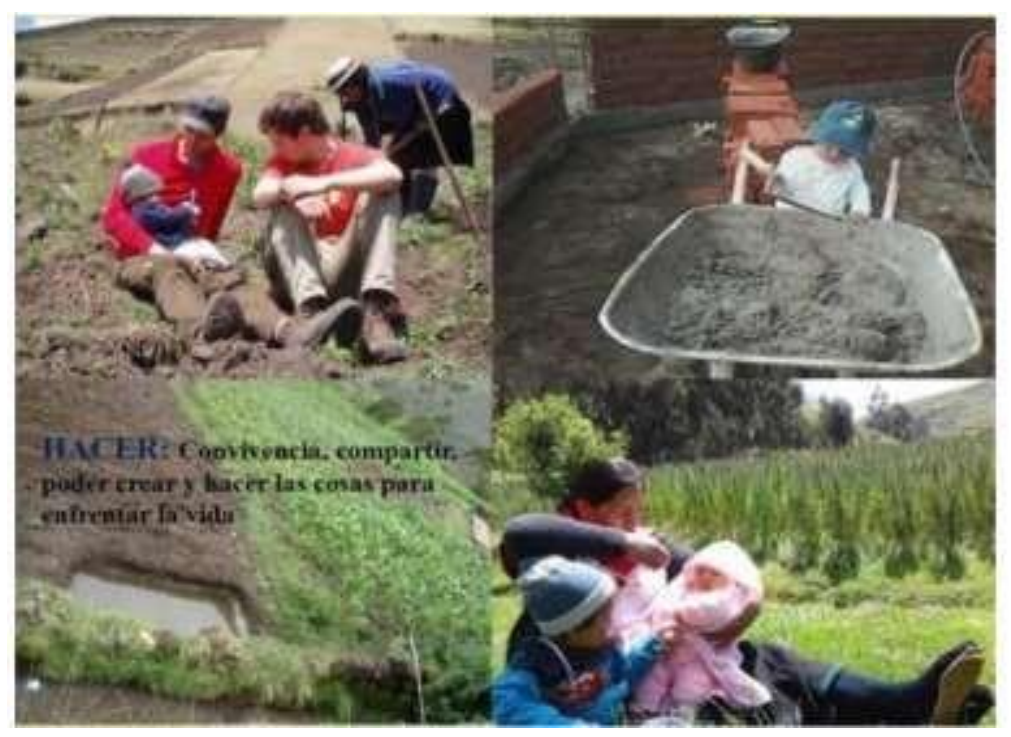

Imagen editada: Bacilio Pomaina Pilamunga

En todas estas fases está presente el: yachag (el saber, conocer, y manejar epistemología), el munay (amar, pasión e intuición por la vida), el ruray (hacer, vivenciar y construir) y ushay (potencia, energía y poder). En base a esta epistemología indígena, se fundamente la estimulación intercultural; los colores, los olores de las plantas, los sonidos de los instrumentos andinos, los juegos, la poesía y entre otros forman parte del saber ancestral cotidiano en la comunidad Ocpote Los Ángeles de Colta Ecuador.

\section{Etapas de estimulación intercultural en el mundo indígena}

La estimulación intercultural en el mundo indígena (Guacho, 2015), consta de tres etapas esencias para la vida: el enamoramiento conocido como pacto, el matrimonio que se consolida con el sirichi (luna de miel indígena), la formación y la enseñanza, del ser humano para la vida; cada una de estas etapas, contiene saberes, códigos culturales y múltiples preceptos de entender y la vida y su entorno. 


\section{Jornada de Investigación Universidad Tecnológica Empresarial de Guayaquil}

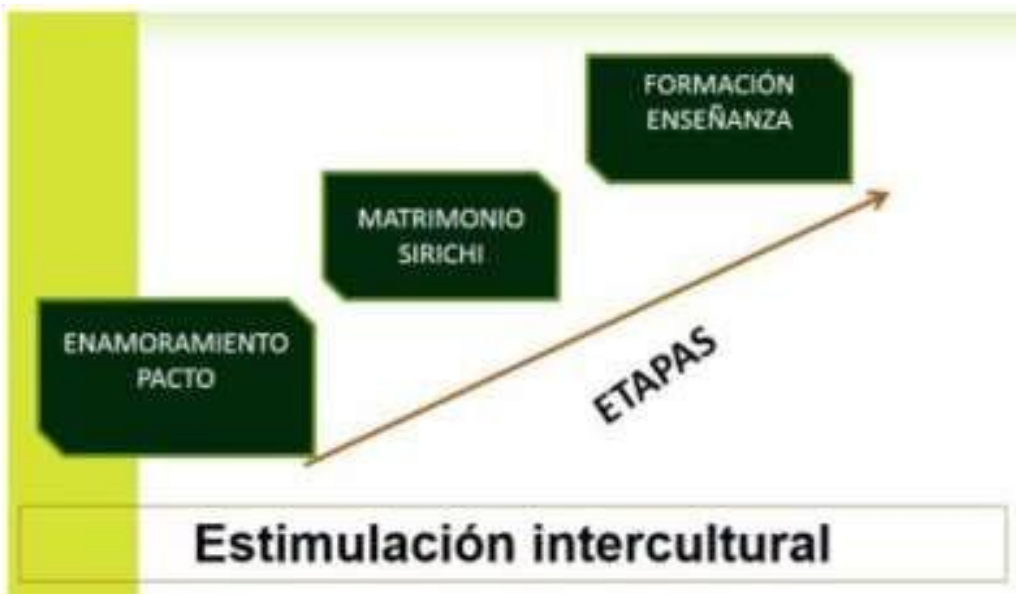

\section{Gráfico 2. Etapas estimulación intercultural}

Fuente: Bacilio Pomaina Pilamunga

Enamoramiento. - Es una época de reencuentro de parejas, un espacio de reflexión y compromiso entre las en las parejas; en estos espacios, se gesta la idea de tener un hijo o hija, como debería es nuevo ser, como criarlo, que nombre ponerlo, cuáles serían los taitas (sabios) mentores, y en el fondo se consolida como un pacto ético cultural de cumplimento para llegar al matrimonio. Este espacio en el pensamiento indígena, es donde se inicia la estimulación, la vida no empieza desde el nacimiento del ser humano sino de mucho más antes de la fecundación.

Matrimonio. - Es el acto oficial público y comunitario de las parejas para dar inicio a ese pacto ético cultural, logrado en el enamoramiento, la cual se consuma con el SIRICHI (luna de miel indígena), es una ceremonia de la unión dual de los novios; por lo tanto el matrimonio es un compromiso de vida dual, unión para la vida, compromiso irrenunciable de separación, aun en épocas difíciles. El yachag (médico o sabio indígena), en una ceremonia comunitaria sierra este pacto y da fe para el cumplimento de las partes, para lo cual encadena a la pareja, con una faja linda de simbología, y acompañada de palabras y consejos de la vida, da por hecho el vínculo conyugal. Ver fotografía.

Formación y enseñanza. - Es una etapa esencial de estimulación intercultural, la cual se parte dando sus primeros estímulos en el vientre materno (canticos, ritos y costumbres culturales), en caso de existir alguna alteración antes del parto, se aplica el manteo (encuadernación de embarazo), el embarazo es monitoreada por la partera, posteriormente la partera se prepara para el parto intercultural, luego de nacer el niño o la niña, se hace el maito (envoltura) y finalmente se entrega a la madre luego de un ritual de intensificación. Ver fotografía. 
Revista Ciencia \& Tecnología No. 16, 31 de octubre de 2017 ISSN impreso: 1390 - 6321

\section{Jornada de Investigación Universidad Tecnológica Empresarial de Guayaquil}

Fotografía 2. Matrimonio

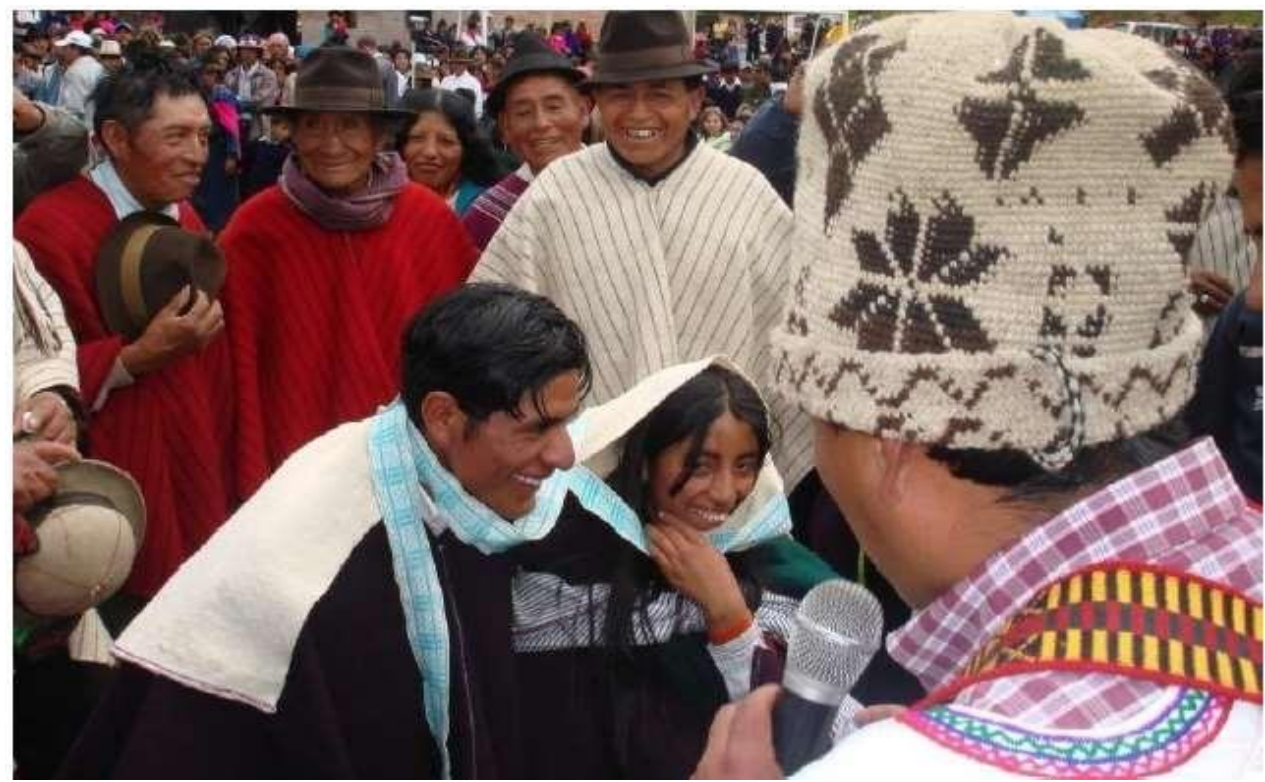

Fotografía matrimonio tomada: 03/16/ 2006

Fotografía 3. Formación y enseñanza

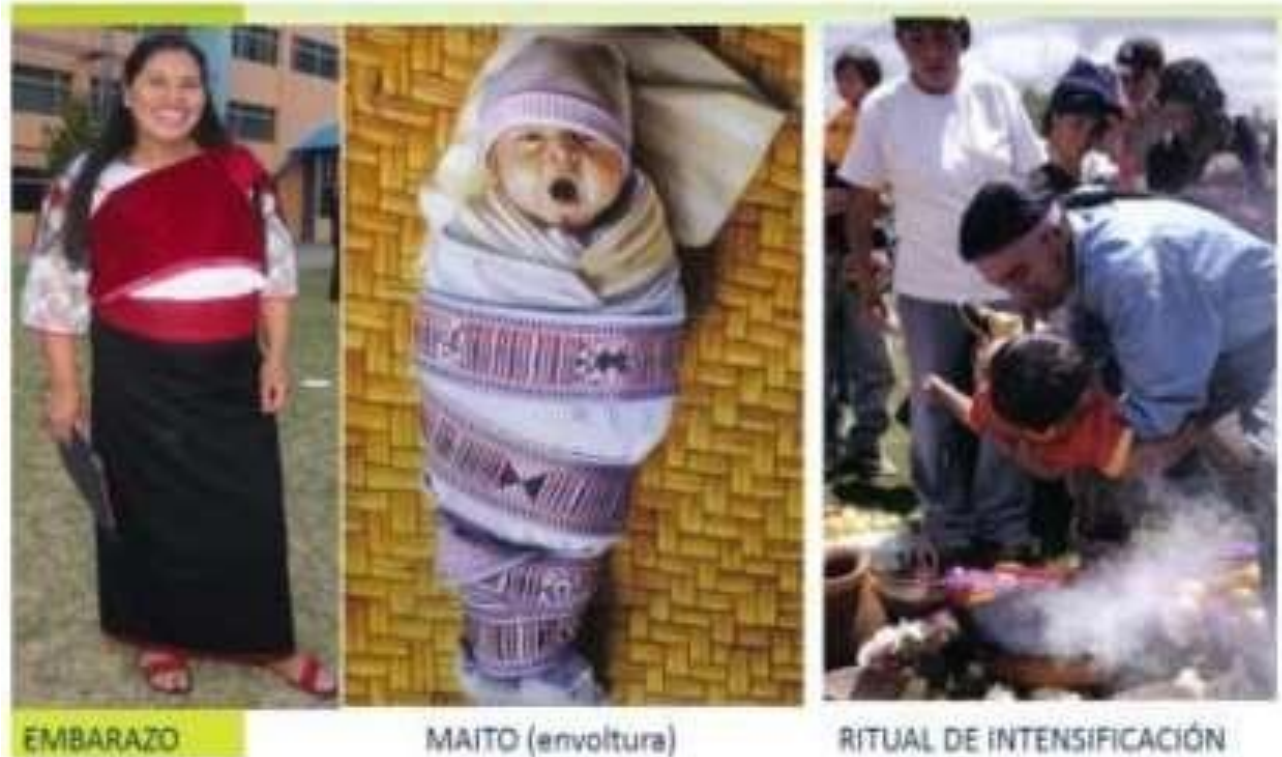

Fotografías editadas: Bacilio Pomaina Pilamunga

Técnicas de estimulación en el mundo indígena

Se define (Balla, 2015) con las metodologías de enseñanza indígena, que se constituyen en las enseñanzas de los ancestrales, a través de prácticas, ritos, 


\section{Jornada de Investigación Universidad Tecnológica Empresarial de Guayaquil}

símbolos y ceremonias; por medio de vías orales (ver, oír, hacer y sentir). Son técnicas metodológicas que divulgan los acontecimientos culturales, históricos, leyes, visiones, preceptos, códigos culturales, poesías y saberes cotidianos de generación en generación. A continuación, se describen las metodologías de enseñanza recopiladas en las comunidades de Ocpotes.

Ritual: son prácticas culturales andinas que se constituyen como las más antiguas ceremonias conocidas y están relacionados con el nacimiento, el matrimonio, la muerte, la sanación y la veneración divina. Estas prácticas se convierten en métodos de enseñanza y aprendizaje para los individuos. A través de estas prácticas se trasmiten valores, significado, símbolos y prácticas cotidianas para el buen vivir y la armonía social.

Recorrido: son vías de acceso por donde se transitan habitualmente los saberes que además constituyen espacios de comunicación entre los seres vivientes, que se evidencian al ver, oír, hacer y sentir. El recorrido es una gira de observación, es una técnica de enseñanza y aprendizaje para los niños, donde se practica el ver, oír y hacer un determinado saber.

Oralidad: son narraciones de acontecimientos históricos, leyes y preceptos, cantos y poemas, sentencias y pensamientos, que han sido transmitidos oralmente de generación en generación en un lugar y espacio preestablecido entre el facilitador y el educando. Es un método de enseñanza y aprendizaje desde la vivencia, practica, preservación, conducta y consejería.

Minga: la minga es una actividad colectiva para cumplir un trabajo o una labor comunitaria; un lugar donde se comparte saberes y conocimientos. Es un espacio colectivo donde se genera un proceso de enseñanza y aprendizaje, en base de la vivencia, práctica y consejería, en cuya actividad se vincula al niño y a la niña.

Imitación: es un proceso mental que permite el aprendizaje a partir de la observación de las actividades de la familia y comunidad; al observar las consecuencias de esa conducta en la supervivencia del otro, al suponer que es un modelo social o incluso para obtener su aprobación o pertenecer a un grupo.

Simbología: es una representación simbólica, que representa la dualidad (representación a dos elementos), tripartición (representación a tres elementos) y la cuatripartición (representación a cuatro elementos); a través de los símbolos se enseña el significado, la connotación y la práctica de los valores ancestrales para la armonía social. En esta práctica está represente el niño y la niña; la cual se evidencia en la vestimenta, en los códigos culturales de las celebraciones y ceremonias.

Dualidad: Hombre - mujer; día - noche, luna - sol; plantas hembras y machos.

Triparticipación: ciclo de la vida humana (nace, desarrolla y muere); dimensión de entender el espacio (cielo, tierra e inframundo).

Cuatripartición: elementos naturales de la vida (agua, aire, fuego y tierra); cuatro celebraciones sagradas (dos solsticios y dos equinoccios). 


\section{Jornada de Investigación Universidad Tecnológica Empresarial de Guayaquil}

\section{Práctica de estimulación intercultural en la cotidianidad}

En las comunidades de Ocpotes aun en la actualidad las madres y los padres, practican los saberes de estimulación intercultural en las hijas e hijos, a continuación, una sistematización de las prácticas más comunes que se usa para educar; las prácticas cotidianas del entorno comunitario, a criterios de los taitas (sabios) y mamas (sabias), se sub-divide tradicionalmente en 4 rango de edades y común mente se usa diferentes técnicas.

0 - a un año: las prácticas más comunes en esta edad es fortalecer la observación y la intuición del niño o de la niña para logra la identificación de objetos o sujetos. Para lo cual se usa la técnica de ritual y recorrido.

Fotografía 5: recorrido en el entorno natural y familiar.

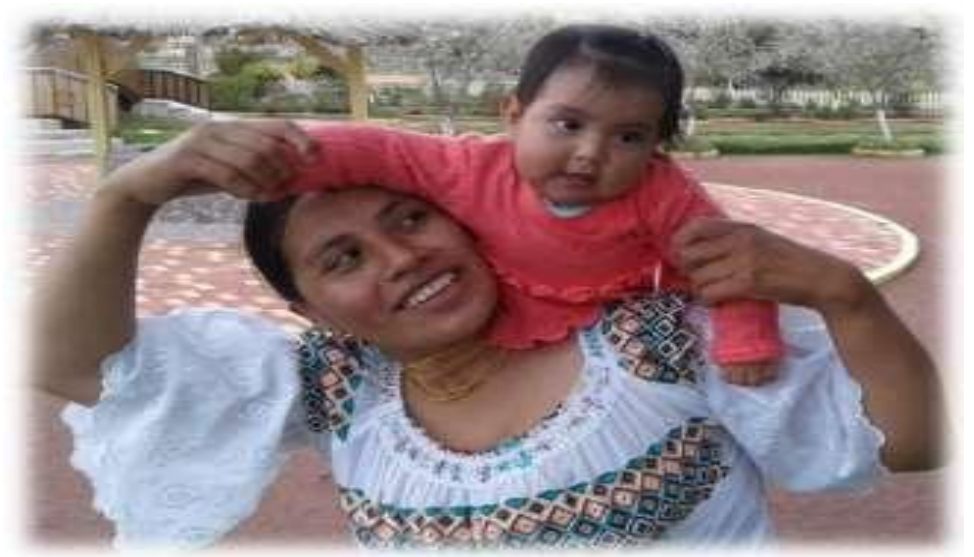

Fotografías editadas: Bacilio Pomaina Pilamunga

1 año en adelante: las prácticas más comunes en esta edad es potenciar la independencia y la acción del niño y de la niña. Para lo cual se usa la técnica de recorrido y oralidad.

\section{Fotografía 6. Conversatorio oral familiar y comunitario}

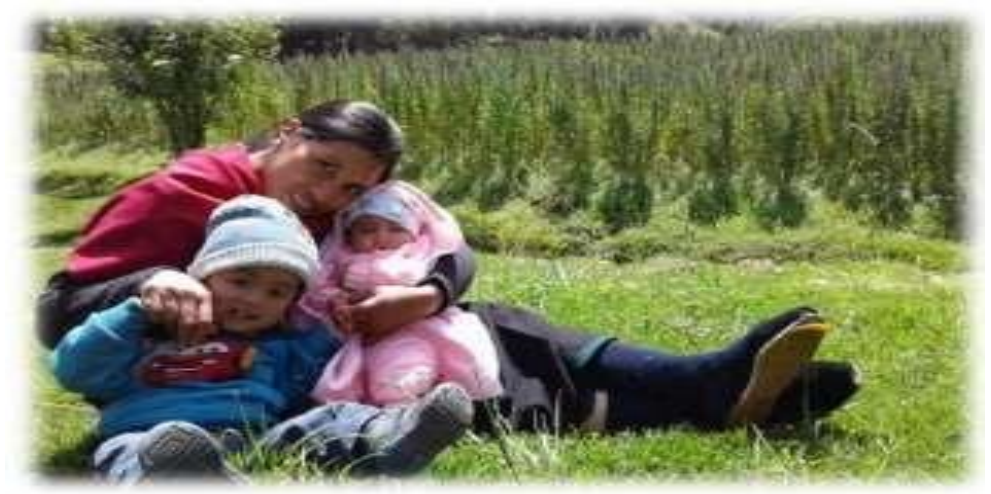

Fotografías editadas: Bacilio Pomaina Pilamunga 


\section{Jornada de Investigación Universidad Tecnológica Empresarial de Guayaquil}

2 años en adelante: las prácticas más comunes en esta edad es desarrollar la habilidad y la imaginación en niño y en la niña. Para lo cual se usa la técnica de oralidad y simbología.

Arte grafica de imaginación y lectura 1. Imaginación de los niños y niñas

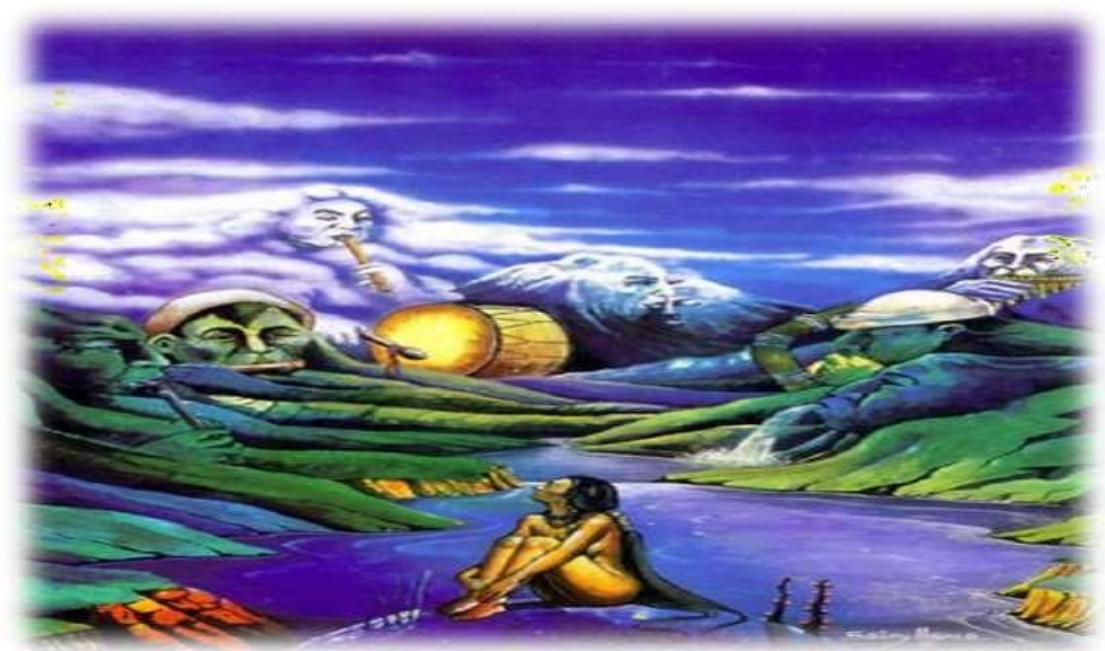

Arte grafica de imaginación y lectura

3 años en adelante: las prácticas más comunes en esta edad es desarrollar y potenciar el hacer para la vida en niño o en la niña. Para lo cual se usa la técnica de imitación y minga. Esta práctica tradicional se ven desde los defensores de los derechos de la niñez, como trabajo infantil o actividad inadecuada; para los actores de esta comunidad es una técnica de aprendizaje que forma a la niñez para enfrentar los restos y los destinos de la vida.

\section{Fotografía 7. Minga de imitación y aprendizaje}

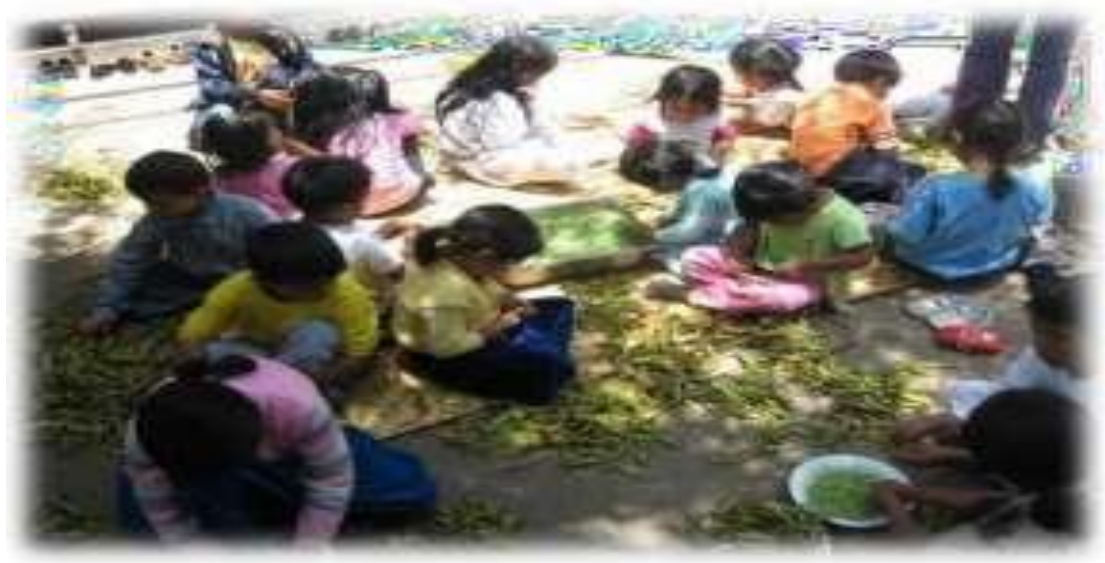

Fotografías editadas: Bacilio Pomaina Pilamunga 


\section{Jornada de Investigación Universidad Tecnológica Empresarial de Guayaquil}

Además de estas prácticas también consideran importante (Comercio, 2014) cambiar de postura al niño o niña cada dos horas (primeros 6 meses), de acuerdo con los puntos de la chakana (cruz andina). El niño en su subconsciente aprende a ubicarse en los cuatro puntos cardinales, que representan a los cuatro elementos sagrados de la vida.

Los colores, los olores de las plantas, los sonidos de los instrumentos andinos y los murmullos de las aves forman parte de las largas horas de juegos que el infante comparte cada día con sus padres, esta practicas permite desarrollar la percepción, inteligencia y motricidad. Además, activan los sentidos como el olfato, el gusto, la vista y el tacto, se convierten en herramientas terapéuticas los instrumentos musicales andinos y las plantas de los huertos.

Las flores de colores vivos y aromas sutiles, son importantes aliadas en el desarrollo del olfato; la cual permite diferenciar los colores y a relacionarlos con la naturaleza; es vital y propicio, hacer pisa descalzo la tierra de la chacra de sus padres, para la conexión con la tierra y donde se adquiere la energía de la Pacha Mama. Estas son las prácticas diarias y cotidianas que aun persiste en la comunidad Ocpote Los Ángeles, lo hace con amor las personas mayores y con una aceptación considerable las jóvenes parejas de la comunidad.

\section{Espacios de estimulación y formación en el mundo indígena}

Enamoramiento-Tupanakuy: es un espacio de encuentro entre un hombre soltero y una mujer soltera para planificar la procreación de la vida. En este encuentro se expresan los sueños, anhelos, la compatibilidad de valores y saberes; en base a esta predicción se fecunda en el pensamiento la gestación de un nuevo ser humano.

Vientre materno-Mamapak Wisha: durante los nueve meses, el nuevo ser aprende y concibe la vida de su madre y su entorno. Es un espacio propicio de enseñanza y aprendizaje de valores, costumbres y visiones, a través de masajes, alocuciones y códigos culturales.

Fogón-Tushpa: es el primer espacio donde el nuevo ser humano aprende a relacionarse e iniciar sus labores de aprendizaje para la vida, el lugar cotidiano de preparación de los alimentos para la familia. El fogón es la primera cuna de enseñanza y aprendizaje del nuevo ser.

Territorio- Kawsana Llacta: es un espacio de enseñanza y aprendizaje donde el ser humano se relaciona con la naturaleza, en este espacio el nuevo ser comienza a amar, respetar y convivir.

Chacra-Murifukuna Ashpa: es entendido como un laboratorio o aula de aprendizaje, donde la persona le hace conocer sus bondades y defectos.

Comunidad- Aylly Llacta: es un espacio comunitario de niños, jóvenes, mujeres y adultos; donde se aprende y cultiva la armonía, la solidaridad, el respeto y la obediencia de unos hacia otros.

Institución Educativa- Yachana Wasi: es una institución oficial de educación y 57 


\section{Jornada de Investigación Universidad Tecnológica Empresarial de Guayaquil}

formación, donde se imparte el razonamiento, la ciencia y los avances tecnológicos para convivir en la vida actual.

En base a estos espacios de aprendizaje, el niño o la niña indígena, no llega a la escuela oficial vacíos de conocimientos y saber, como ha considerado la ciencia oficial.

\section{Áreas de estimulación y formación en el mundo indígena}

La educación andina (Guacho, 2015) siempre existió en las comunidades indígenas, constituyendo uno de los pilares de la vida, con sus propias metodologías y espacios, que convivían en un territorio, producían sus productos, bajo normas y autoridades, que jamás fueron reconocidas en los estamentos del estado colonial. A continuación, se describen las principales áreas de formación ancestral que aún prevalecen en la terminología actual:

Territorio: es un área de conocimiento sobre el ordenamiento natural, lugares sagrados, hidrografía, ecosistemas y sus conceptos para entender la pachamama y su correlación; el estudiante andino debe poseer estos conocimientos para enfrentar la vida y sus desafíos.

Soberanía alimentaria: es un área de conocimiento que debe poseer un hombre o mujer andino sobre cultivos, semillas, valor cultural, gastronomía, técnica de producción agroecológica y las bondades nutricionales para la convivencia humana.

Gobierno y administración: es un área de conocimiento sobre Derecho mayor, administración comunitaria, derechos colectivos, ética colectiva y justicia indígena con su legado humano y natural, que son bases con las que debe formarse un nuevo ser andino.

Desarrollo: es un área de conocimiento sobre visión comunitaria, plan de vida y comercio justo que alimente al ser humano, a la comunidad y a la pachamama; una visión integral y global en que se debe educar el individuo andino.

Medicina: es una rama de conocimiento sobre un sistema alternativo de medicina que armoniza el interior del ser humano y sus correlaciones con el alma, espíritu y cuerpo; el estudiante andino debe poseer estos conocimientos para vivir una vida de paz y armonía.

Población: es un área de conocimiento sobre el origen, historia, mitología, poesía, indumentaria, lingüística y las formas de vida que posee cada pueblo o cultura. La mujer $y$ el hombre andino deben poseer estos conocimientos para fortalecer $y$ potenciar su cultura.

\section{Niveles de educación en el mundo indígena}

Así como la educación tradicional oficial (Guacho, 2015) posee sus niveles de formación, en el mundo indígena también existen varios niveles. El artículo en mención es una parte de la formación considerada la estimulación intercultural, el aprendizaje y la enseñanza, en el mundo indígena no se concluye, es un proceso permanente de aprendizaje, se concluye cuando, cuando se va de este mundo. A continuación, los niveles de educación en el mundo indígena. 


\section{Jornada de Investigación Universidad Tecnológica Empresarial de Guayaquil}

Wawakuna (niñez): en este nivel es propicia la estimulación intercultural que va desde 0 años hasta 12 años, la educación se centra en aprendizaje para la vida desde la vida.

Wambrakuna (adolescencia y juventud): en este nivel es propicia la formación intelectual que va desde 12 años hasta logar el matrimonio, la educación se centra en el experimento y acumulación de experiencias desde la vida.

Runa (personas adultas): en este nivel es propicia la aplicación intelectual de lo aprendido, que va desde el matrimonio hasta ocupar el primer cargo comunal, social o político; la educación se centra en ser guía de experiencias desde sus vivencias vividas.

Taitas (sabios): es un nivel máximo que se puede alcanzar el runa, por lo general a este nivel llegan personas pasadas de 45 años de esta, quienes son, sabios, guiadores y aconsejadores de experiencias de vidas. A estas personas sabias acuden Wambrakuna y runa para que les enseñen; son respetados por la comunidad, poseedores de grandes saberes intelectuales, que la comunidad asido beneficiaria.

\section{Conclusiones}

En nuestro recorrido, al vivenciar la práctica de los saberes ancestrales; se afirma que la estimulación intercultural es un método de formación para la vida al ser humano, esto implica la relación o conexión con las cuatro comunidades (natural, humana, divino y ancestral) de entorno en un vínculo integral, y por lo tanto es un derecho a la educación irrenunciable, pero esa formación no es solo educar en área secular, sino en contexto integral para la convivencia mutua.

La estimulación es apenas el inicio del proceso formativo indígena. Al recopilar y sistematizar los saberes de la estimulación intercultural, se evidencia como un eje primordial, que se convierte en un camino para recuperar la cultura, la historia, los códigos culturales y los espacios de educación propia, que se basa en la sabiduría, conocimientos y ciencia local. En los términos actuales también se puede considerar, como la pedagogía de enseñanza indígena, que en los actuales tiempos, se ha transformado en lo holístico, integral, endógeno en relación al diversidad; la cual busca logar la transformación en una sociedad diversa, pluralista y ética.

En el estado plurinacional del Ecuador, es evidente el reconocimiento del derecho de los pueblos indígenas y sus formas de vida, están presente en los instrumentos jurídicos nacionales e internacionales; pero en la consecución práctica la educación y la enseñanza indígena tan solo siguen siendo una iniciativa local indígena.

\section{Referencias bibliográficas}

Balla, M. (6 de agosto de 2015). Estimulación Intercultural. (B. Pomaina, Entrevistador)

Cachiguango, E. (2007). Educación Indígena. Otavalo Ecuador: Escuela de Gobierno.

Cepeda, M. (7 de agosto de 2015). Enseñanza a la niñez. (B. Pomaina, Entrevistador) 


\section{Jornada de Investigación Universidad Tecnológica Empresarial de Guayaquil}

Comercio, E. (8 de octubre de 2014). Estimulación intercultural. Riobamba, Chimborazo, Ecuador.

Guacho, A. M. (4 de agosto de 2015). Estimulación Intercultural. (B. Pomaina, Entrevistador).

Mullo, M. Y. (7 de agosto de 2015). Educación Indígena. (B. Pomaina, Entrevistador). 